\title{
NEW IDENTITIES FOR RAMANUJAN'S CUBIC CONTINUED FRACTION
}

\author{
M.S. Mahadeva Naika, S. Chandankumar, K. Sushan Bairy
}

\begin{abstract}
In this paper, we present some new identities providing relations between Ramanujan's cubic continued fraction $V(q)$ and the other three continued fractions $V\left(q^{9}\right), V\left(q^{17}\right)$ and $V\left(q^{19}\right)$. In the process, we establish some new modular equations for the ratios of Ramanujan's theta functions. We also establish some general formulas for the explicit evaluations of ratios of Ramanujan's theta functions.
\end{abstract}

Keywords: Cubic continued fraction, Modular equation, Theta-function

\section{Introduction}

In Chapter 16, of his second notebook [13], [5, pp.257-262], S. Ramanujan develops the theory of theta function and his theta function is defined by

$$
\begin{aligned}
f(a, b) & :=\sum_{n=-\infty}^{\infty} a^{n(n+1) / 2} b^{n(n-1) / 2}, \quad|a b|<1, \\
& =(-a ; a b)_{\infty}(-b ; a b)_{\infty}(a b ; a b)_{\infty},
\end{aligned}
$$

where, $(a ; q)_{\infty}:=\prod_{n=1}^{\infty}\left(1-a q^{n-1}\right),|q|<1$.

Research supported by UGC, Government of India, New Delhi under major research project No.F.No.34-140\2008 (SR).

2010 Mathematics Subject Classification: primary: 33D10; secondary: 11A55, 11F27 
Following Ramanujan, we define

$$
\begin{aligned}
\varphi(q) & :=f(q, q)=\sum_{n=-\infty}^{\infty} q^{n^{2}}=\left(-q ; q^{2}\right)_{\infty}^{2}\left(q^{2} ; q^{2}\right)_{\infty}, \\
\psi(q) & :=f\left(q, q^{3}\right)=\sum_{n=0}^{\infty} q^{n(n+1) / 2}=\frac{\left(q^{2} ; q^{2}\right)_{\infty}}{\left(q ; q^{2}\right)_{\infty}} \\
f(-q) & :=f\left(-q,-q^{2}\right)=\sum_{n=-\infty}^{\infty}(-1)^{n} q^{n(3 n-1) / 2}=(q ; q)_{\infty} .
\end{aligned}
$$

On page 366 of his 'lost' notebook [14], Ramanujan has recorded cubic continued fraction as follows:

$$
V(q):=\frac{q^{1 / 3}}{1}+\frac{q+q^{2}}{1}+\frac{q^{2}+q^{4}}{1}+\frac{q^{3}+q^{6}}{1}+\cdots, \quad|q|<1,
$$

and asserted by stating "and many results analogous to the previous continued fraction." Motivated by this fact, H. H. Chan [7] developed the theory of the cubic continued fraction and established relations between $V(q)$ and other three continued fractions $V(-q), V\left(q^{2}\right)$ and $V\left(q^{3}\right)$. In [3], N. D. Baruah established modular equations connecting $V(q)$ with $V\left(q^{5}\right)$ and $V\left(q^{7}\right)$ respectively. In [9], M. S. Mahadeva Naika established several results of cubic continued fraction which are analogous to Rogers-Ramanujan continued fraction [13]. In [8], B. Cho, J. K. Koo and Y. K. Park have extended the results of Chan [7] and Baruah [3] and established relations connecting $V(q)$ with $V\left(q^{11}\right), V\left(q^{13}\right)$ and $V\left(q^{17}\right)$ respectively. In [17], J. Yi and H. S. Sim established some new modular equations by employing the theory of modular forms. For more details on Ramanujan's cubic continued fraction, one can see [1], [2], [11]. Motivated by these works, in this paper, we establish some new modular equations connecting $V(q)$ with $V\left(q^{9}\right), V\left(q^{17}\right)$ and $V\left(q^{19}\right)$ respectively.

In [15], Yi introduced two parameterizations $h_{k, n}$ and $h_{k, n}^{\prime}$ as follows:

$$
\begin{aligned}
h_{k, n} & :=\frac{\varphi\left(e^{-\pi \sqrt{n / k}}\right)}{k^{1 / 4} \varphi\left(e^{-\pi \sqrt{n k}}\right)}, \\
h_{k, n}^{\prime} & :=\frac{\varphi\left(-e^{-\pi \sqrt{n / k}}\right)}{k^{1 / 4} \varphi\left(-e^{-\pi \sqrt{n k}}\right)}
\end{aligned}
$$

and established several properties as well as explicit evaluations of $h_{k, n}$ and $h_{k, n}^{\prime}$ for different positive rational values of $n$ and $k$.

In [4], Baruah and Nipen Saikia and [16] Yi, Y. Lee and D. H. Paek have defined two parameters $l_{k, n}$ and $l_{k, n}^{\prime}$ as follows:

$$
l_{k, n}:=\frac{\psi\left(-e^{-\pi \sqrt{n / k}}\right)}{k^{1 / 4} e^{-\frac{(k-1) \pi}{8} \sqrt{n / k}} \psi\left(-e^{-\pi \sqrt{n k}}\right)},
$$


and

$$
l_{k, n}^{\prime}:=\frac{\psi\left(e^{-\pi \sqrt{n / k}}\right)}{k^{1 / 4} e^{-\frac{(k-1) \pi}{8} \sqrt{n / k}} \psi\left(e^{-\pi \sqrt{n k}}\right)} .
$$

They have also established several properties as well as explicit evaluations of $l_{k, n}$ and $l_{k, n}^{\prime}$ for different positive rational values of $n$ and $k$. In [12], Mahadeva Naika, K. Sushan Bairy and M. Manjunatha have established several new modular equations of degree 4 and established general formulas for explicit evaluations of $h_{4, n}$. In [10], Mahadeva Naika, S. Chandankumar and Bairy have established several new modular equations of degree 9, and also established several general formulas for the explicit evaluations of $l_{9, n}$ and $l_{9, n}^{\prime}$.

In Section 3, we establish some new modular equations for the ratios of Ramanujan's theta function. In Section 4, we establish some general formulas for the explicit evaluations of ratios of Ramanujan's theta functions by using the results obtained in Section 3. In Section 5, we establish some new relations connecting $V(q)$ with other three continued fractions $V\left(q^{9}\right), V\left(q^{17}\right)$ and $V\left(q^{19}\right)$ respectively.

\section{Preliminary results}

In this section, we collect some relevant identities that are useful in proving our main results.

Lemma 2.1 ([5, Ch.20, Entry 1(iii), p. 345]). We have

$$
\begin{aligned}
& \frac{\varphi\left(q^{1 / 3}\right)}{\varphi\left(q^{3}\right)}=1+\left(\frac{\varphi^{4}(q)}{\varphi^{4}\left(q^{3}\right)}-1\right)^{1 / 3} \\
& \frac{3 \varphi\left(q^{9}\right)}{\varphi(q)}=1+\left(\frac{9 \varphi^{4}\left(q^{3}\right)}{\varphi^{4}(q)}-1\right)^{1 / 3}
\end{aligned}
$$

Lemma 2.2. If $P=\frac{\varphi(q)}{\varphi\left(q^{9}\right)}$ and $Q=\frac{\varphi\left(q^{3}\right)}{\varphi\left(q^{27}\right)}$, then

$$
\left(3-P-\frac{3}{P}\right)\left(3-Q-\frac{3}{Q}\right)=\left(\frac{Q}{P}\right)^{2} .
$$

Proof. Using equations (2.1) and (2.2), we arrive at the equation (2.3).

Lemma $2.3\left(\left[17\right.\right.$, Throrem 2.1]). Let $P=\frac{f(-q)}{q^{1 / 12} f\left(-q^{3}\right)}$ and $Q=\frac{f\left(-q^{17}\right)}{q^{17 / 12} f\left(-q^{51}\right)}$, 
then

$$
\begin{aligned}
& \left(\frac{Q}{P}\right)^{9}-\left(\frac{P}{Q}\right)^{9}-238\left[\left(\frac{Q}{P}\right)^{6}+\left(\frac{P}{Q}\right)^{6}\right]+1853\left[\left(\frac{Q}{P}\right)^{3}-\left(\frac{P}{Q}\right)^{3}\right] \\
& -17\left(\frac{Q^{8}}{P^{4}}+\frac{3^{2} P^{4}}{Q^{8}}\right)-17\left(\frac{3^{2} Q^{4}}{P^{8}}+\frac{P^{8}}{Q^{4}}\right)+34\left(Q^{7} P-\frac{3^{4}}{Q^{7} P}\right) \\
& -34\left(Q P^{7}-\frac{3^{4}}{Q P^{7}}\right)-442\left(\frac{Q^{5}}{P}-\frac{3^{2} P}{Q^{5}}\right)-442\left(\frac{3^{2} Q}{P^{5}}-\frac{P^{5}}{Q}\right) \\
= & (P Q)^{8}+\left(\frac{3}{P Q}\right)^{8}-34\left[(P Q)^{6}+\left(\frac{3}{P Q}\right)^{6}\right] \\
& +425\left[(P Q)^{4}+\left(\frac{3}{P Q}\right)^{4}\right]-2380\left[(P Q)^{2}+\left(\frac{3}{P Q}\right)^{2}\right]+8568 .
\end{aligned}
$$

Lemma 2.4 ([17, Theorem 2.3]). Let

$$
P=\frac{f(-q)}{q^{1 / 12} f\left(-q^{3}\right)} \quad \text { and } \quad Q=\frac{f\left(-q^{19}\right)}{q^{19 / 12} f\left(-q^{57}\right)},
$$

then

$$
\begin{aligned}
& \left(\frac{Q}{P}\right)^{10}-\left(\frac{P}{Q}\right)^{10}-76\left[\left(\frac{Q}{P}\right)^{8}-\left(\frac{P}{Q}\right)^{8}\right]+912\left(\left(\frac{Q}{P}\right)^{6}-\left(\frac{P}{Q}\right)^{6}\right) \\
& +6650\left[\left(\frac{Q}{P}\right)^{4}-\left(\frac{P}{Q}\right)^{4}\right]+9481\left[\left(\frac{Q}{P}\right)^{2}-\left(\frac{P}{Q}\right)^{2}\right]-570\left(\frac{3^{3} Q}{P^{7}}+\frac{P^{7}}{Q}\right) \\
& -19\left(\frac{Q^{9}}{P^{3}}+\frac{3^{3} P^{3}}{Q^{9}}\right)-19\left(\frac{3^{3} Q^{3}}{P^{9}}+\frac{P^{9}}{Q^{3}}\right)-570\left(\frac{Q^{7}}{P}+\frac{3^{3} P}{Q^{7}}\right) \\
& +19\left(Q^{8} P^{4}-\frac{3^{6}}{Q^{8} P^{4}}\right)-19\left(Q^{4} P^{8}-\frac{3^{6}}{Q^{4} P^{8}}\right)-2166\left(Q^{5} P+\frac{3^{3}}{Q^{5} P}\right) \\
& -2166\left(Q P^{5}+\frac{3^{3}}{Q P^{5}}\right)=(P Q)^{9}+\left(\frac{3}{P Q}\right)^{9}+3211\left[(P Q)^{3}+\left(\frac{3}{P Q}\right)^{3}\right] .
\end{aligned}
$$

Lemma 2.5 ([5, Ch. 16, Entry 24(ii), p. 39]). We have

$$
f^{3}(-q)=\varphi^{2}(-q) \psi(q) .
$$

Lemma 2.6 ([5, Ch. 20, Entry 1 (i), p. 345]). We have

$$
1+\frac{1}{V(q)}=\frac{\psi^{4}(q)}{q \psi^{4}\left(q^{3}\right)}
$$

where $V(q)$ is defined as in the equation (1.4). 
Lemma 2.7 ([1, Theorem 5.1]). If $P=\frac{\psi(-q)}{q^{1 / 4} \psi\left(-q^{3}\right)} \quad$ and $\quad Q=\frac{\varphi(q)}{\varphi\left(q^{3}\right)}$, then

$$
Q^{4}+P^{4} Q^{4}=9+P^{4} \text {. }
$$

Lemma 2.8 ([15, Theorem 2.2(ii)]). We have

$$
h_{k, n} h_{k, 1 / n}=1 \text {. }
$$

\section{New modular equations for the ratios of Ramanujan's theta functions}

In this section, we establish some new modular equations of degree 3 for the ratios of Ramanujan's theta functions.

Theorem 3.1. If $P=\frac{\varphi(q)}{\varphi\left(q^{3}\right)}$ and $Q=\frac{\varphi\left(q^{9}\right)}{\varphi\left(q^{27}\right)}$, then

$$
\begin{aligned}
& \frac{Q^{5}}{P^{5}}+9\left(\frac{Q^{3}}{P^{3}}-\frac{Q^{4}}{P^{4}}\right)+9\left(\frac{5 Q}{P}+\frac{9 P}{Q}\right)+9\left(Q^{4}+\frac{9}{P^{4}}\right) \\
& +3\left(Q^{2}+3 P^{2}\right)\left(P Q+\frac{9}{P^{3} Q^{3}}\right) \\
= & 135+3\left(\frac{Q^{2}}{P^{2}}+\frac{9 P^{2}}{Q^{2}}\right)+\left(P^{4} Q^{4}+\frac{3^{4}}{P^{4} Q^{4}}\right) \\
& +9\left(P^{2} Q^{2}+\frac{3^{2}}{P^{2} Q^{2}}\right) .
\end{aligned}
$$

Proof. Equations (2.1) and (2.2) can be written as,

$$
\begin{aligned}
& \frac{\varphi\left(q^{3}\right)}{\varphi\left(q^{27}\right)}=1+\left(\frac{\varphi^{4}\left(q^{9}\right)}{\varphi^{4}\left(q^{27}\right)}-1\right)^{1 / 3}, \\
& \frac{3 \varphi\left(q^{9}\right)}{\varphi(q)}=1+\left(\frac{9 \varphi^{4}\left(q^{3}\right)}{\varphi^{4}(q)}-1\right)^{1 / 3} .
\end{aligned}
$$

Using the above equation (3.2) in the equation (2.3), we deduce that

$$
\begin{aligned}
3 n P^{4}-3 P^{4} & +6 n^{2} m P^{4}-3 m^{2} P^{4}+6 n m^{2} P^{4}-3 n^{2} P^{4} \\
& +3 m P^{4}+3 Q^{4}+m^{2} P^{4} Q^{4}+m P^{4} Q^{4}+9 n^{2}+9 n=0,
\end{aligned}
$$

where $m=\left(\frac{9}{P^{4}}-1\right)^{1 / 3}$ and $n=\left(Q^{4}-1\right)^{1 / 3}$.

Solving for $m$ and then cubing both sides, we deduce that

$$
\begin{aligned}
& 729 P^{4}+81 Q^{4}+81 n^{2}+81 n-729 n P^{4}+729 n^{2} P^{4}-999 Q^{4} P^{4} \\
& +30 Q^{8} n^{2} P^{4}+42 n P^{4} Q^{8}+27 Q^{4} n^{2} P^{4}+P^{12} Q^{8} n^{2}+5 P^{12} n Q^{8} \\
& -12 P^{8} n^{2} Q^{8}-51 P^{8} Q^{8} n+9 n^{2} P^{12} Q^{4}-108 n^{2} Q^{4}-54 Q^{4} n-Q^{12} \\
& -9 n^{2} Q^{8}-36 Q^{8} n+10 P^{12} Q^{8}-111 Q^{8} P^{8}-9 P^{12} Q^{4}-81 Q^{8} \\
& +108 P^{8} Q^{4}+486 n P^{4} Q^{4}+273 P^{4} Q^{8}-108 P^{8} Q^{4} n^{2}=0 .
\end{aligned}
$$


Again, solving for $n$ and then cubing both sides, we deduce that

$$
\begin{aligned}
& 81 P+81 P^{3} Q^{2}-81 Q^{4} P+81 P^{6} Q^{3}+135 P^{5} Q^{4}+Q^{8} P^{9}+81 P^{4} Q \\
& +27 P^{2} Q^{3}+Q^{9}-9 Q^{8} P^{5}+27 P^{7} Q^{2}+45 P^{4} Q^{5}+3 Q^{6} P^{3}+9 P^{2} Q^{7} \\
& \left.+9 P^{7} Q^{6}+9 P Q^{8}+9 P^{8} Q^{5}+3 P^{6} Q^{7}\right) \\
\times & \left(81 P-81 Q^{4} P-81 P^{6} Q^{3}+135 P^{5} Q^{4}+Q^{8} P^{9}-81 P^{4} Q-27 P^{2} Q^{3}\right. \\
& -Q^{9}-9 Q^{8} P^{5}+27 P^{7} Q^{2}+81 P^{3} Q^{2}-45 P^{4} Q^{5}+3 Q^{6} P^{3}-9 P^{2} Q^{7} \\
& \left.+9 P^{7} Q^{6}+9 P Q^{8}-9 P^{8} Q^{5}-3 P^{6} Q^{7}\right) \\
& \times\left(6561 P^{2}-13122 P^{2} Q^{4}+24057 P^{6} Q^{4}-26730 Q^{8} P^{6}-810 P^{12} Q^{10}\right. \\
& +8019 Q^{8} P^{2}+Q^{18}-1512 Q^{12} P^{2}+63 P^{2} Q^{16}+729 P^{14} Q^{4} \\
& +11745 P^{10} Q^{8}+2925 P^{6} Q^{12}-8748 P^{10} Q^{4}-2970 Q^{12} P^{10}-972 P^{14} Q^{8} \\
& +297 P^{14} Q^{12}-168 P^{6} Q^{16}-18 P^{14} Q^{16}+99 P^{10} Q^{16}+P^{18} Q^{16} \\
& -324 Q^{10} P^{4}+117 Q^{14} P^{4}+5265 P^{8} Q^{10}-36 P^{8} Q^{14}+2187 P^{8} Q^{2} \\
& +729 P^{12} Q^{6}+13365 Q^{6} P^{4}+165 P^{12} Q^{14}+27 P^{16} Q^{10}-18 P^{16} Q^{14} \\
& \left.-7290 P^{8} Q^{6}-13122 P^{4} Q^{2}\right)=0 .
\end{aligned}
$$

By examining the behavior of the above factors near $q=0$, we can find a neighborhood about the origin, where the second factor is zero; whereas other factors are not zero in this neighborhood. By the Identity Theorem the second factor vanishes identically. This completes the proof.

Remark 1. The equation (3.1) holds for $P=\frac{\psi(q)}{q^{1 / 4} \psi\left(q^{3}\right)}$ and $Q=\frac{\psi\left(q^{9}\right)}{q^{9 / 4} \psi\left(q^{27}\right)}$.

Theorem 3.2. If $P=\frac{\psi(q) \psi\left(q^{17}\right)}{q^{9 / 2} \psi\left(q^{3}\right) \psi\left(q^{51}\right)}$ and $Q=\frac{\psi(q) \psi\left(q^{51}\right)}{q^{-4} \psi\left(q^{3}\right) \psi\left(q^{17}\right)}$, then

$$
\begin{aligned}
& Q^{9}-\frac{1}{Q^{9}}+34\left(Q^{8}+\frac{1}{Q^{8}}\right)+272\left(Q^{7}-\frac{1}{Q^{7}}\right)+238\left(Q^{6}+\frac{1}{Q^{6}}\right) \\
& -595\left(Q^{5}-\frac{1}{Q^{5}}\right)-510\left(Q^{4}+\frac{1}{Q^{4}}\right)+16303\left(Q^{3}-\frac{1}{Q^{3}}\right) \\
& -5202\left(Q^{2}+\frac{1}{Q^{2}}\right)-26911\left(Q-\frac{1}{Q}\right)+\left(P^{8}+\frac{3^{8}}{P^{8}}\right)+20230 \\
= & 17\left\{( P ^ { 2 } + \frac { 3 ^ { 2 } } { P ^ { 2 } } ) \left[7\left(Q^{6}+\frac{1}{Q^{6}}\right)+28\left(Q^{5}-\frac{1}{Q^{5}}\right)+34\left(Q^{4}+\frac{1}{Q^{4}}\right)\right.\right. \\
& \left.-168\left(Q^{3}-\frac{1}{Q^{3}}\right)+160\left(Q^{2}+\frac{1}{Q^{2}}\right)+378\left(Q-\frac{1}{Q}\right)-210\right] \\
& -\left(P^{4}+\frac{3^{4}}{P^{4}}\right)\left[5\left(Q^{4}+\frac{1}{Q^{4}}\right)+21\left(Q^{3}-\frac{1}{Q^{3}}\right)-35\left(Q^{2}+\frac{1}{Q^{2}}\right)\right. \\
& \left.\left.-14\left(Q-\frac{1}{Q}\right)-30\right]-\left(P^{6}+\frac{3^{6}}{P^{6}}\right)\left[\left(Q^{2}+\frac{1}{Q^{2}}\right)-2\left(Q-\frac{1}{Q}\right)-2\right]\right\} .
\end{aligned}
$$


Proof. Using the equation (2.6) in the equation (2.4), we find that

$$
\begin{aligned}
& 238 c^{5} d^{5} a b+238 a^{5} b^{5} c d-1853 c^{4} d^{4} a^{2} b^{2}+1853 a^{4} b^{4} c^{2} d^{2}+8568 a^{3} b^{3} c^{3} d^{3} \\
& -34 a^{5} b^{5} c^{5} d^{5}-24786 a b c d+2754 a^{2} b^{2} p^{2} p_{1}^{2}+153 a^{4} b^{4} p p_{1}+153 c^{4} d^{4} p_{1} p \\
& -2754 p^{2} c^{2} d^{2} p_{1}^{2}+6561 p p_{1}+442 c^{4} d^{4} p_{1}^{2} a^{2} b^{2} p^{2}-442 a^{4} b^{4} p^{2} c^{2} d^{2} p_{1}^{2} \\
& -3978 a^{3} b^{3} p c d p_{1}+3978 c^{3} d^{3} p_{1} a b p+17 c^{5} d^{5} p_{1}^{2} a b p^{2}+17 a^{5} b^{5} p^{2} c d p_{1}^{2} \\
& -21420 a^{2} b^{2} p c^{2} d^{2} p_{1}-2380 a^{3} b^{3} p^{2} c^{3} d^{3} p_{1}^{2}-34 c^{5} d^{5} p_{1} a^{3} b^{3} p+34 c^{3} d^{3} p_{1} a^{5} b^{5} p \\
& +425 a^{4} b^{4} p c^{4} d^{4} p_{1}+34425 a b p^{2} c d p_{1}^{2}-c^{6} d^{6}+a^{6} b^{6}+a^{5} b^{5} p^{2} c^{5} d^{5} p_{1}^{2}=0
\end{aligned}
$$

where

$$
\begin{aligned}
& a=\frac{\varphi^{2}(-q)}{\varphi^{2}\left(-q^{3}\right)}, \quad b=\frac{\psi(q)}{q^{1 / 4} \psi\left(q^{3}\right)}, \quad c=\frac{\varphi^{2}\left(-q^{17}\right)}{\varphi^{2}\left(-q^{51}\right)}, \\
& d=\frac{\psi\left(q^{17}\right)}{q^{17 / 4} \psi\left(q^{51}\right)}, \quad p=\frac{f(-q)}{q^{1 / 12} f\left(-q^{3}\right)}, \quad p_{1}=\frac{f\left(-q^{17}\right)}{q^{17 / 12} f\left(-q^{51}\right)} .
\end{aligned}
$$

Collecting the terms containing $p p_{1}$ on one side of the equation (3.7) and then cubing both sides, we deduce that

$$
\begin{aligned}
& 714 c_{2}^{8} c d^{17} a b+714 a_{2}^{8} a b^{17} c d+19758444939 c_{2}^{2} c d^{5} a b+19758444939 a_{2}^{2} a b^{5} c d \\
& +2243133 a b c_{2}^{6} c d^{13}+2243133 a_{2}^{6} a b^{13} c d+406552365 a_{2}^{4} a b^{9} c d+406552365 c_{2}^{4} c d^{9} a b \\
& -31093 a_{2}^{5} b^{10} c_{2}^{8} d^{16}+55006404858 c_{2}^{4} d^{8} a_{2}^{3} b^{6}-420929877 c_{2}^{7} d^{14} a_{2}^{2} b^{4} \\
& -9563678423 c_{2}^{6} d^{12} a_{2}^{3} b^{6}-167688 c_{2}^{8} d^{16} a_{2} b^{2}+420929877 a_{2}^{7} b^{14} c_{2}^{2} d^{4} \\
& +167688 a_{2}^{8} b^{16} c_{2} d^{2}+141404912 a_{2}^{7} b^{14} c_{2}^{4} d^{8}+3008487501 a_{2}^{3} b^{6} c_{2}^{2} d^{4} \\
& +31093 a_{2}^{8} b^{16} c_{2}^{5} d^{10}-821508 a_{2}^{8} b^{16} c_{2}^{3} d^{6}-598879332 a_{2}^{6} b^{12} c_{2} d^{2} \\
& +103084180848 a_{2}^{5} b^{10} c_{2}^{2} d^{4}+5661 a_{2}^{7} b^{14} c_{2}^{6} d^{12}+13164103527 c_{2}^{5} d^{10} a_{2}^{4} b^{8} \\
& -13164103527 c_{2}^{4} d^{8} a_{2}^{5} b^{10}+9563678423 c_{2}^{3} d^{6} a_{2}^{6} b^{12}-141404912 c_{2}^{7} d^{14} a_{2}^{4} b^{8} \\
& +75454602 c_{2}^{6} d^{12} a_{2}^{5} b^{10}+821508 c_{2}^{8} d^{16} a_{2}^{3} b^{6}-75454602 c_{2}^{5} d^{10} a_{2}^{6} b^{12} \\
& +598879332 c_{2}^{6} d^{12} a_{2} b^{2}-55006404858 c_{2}^{3} d^{6} a_{2}^{4} b^{8}-103084180848 c_{2}^{5} d^{10} a_{2}^{2} b^{4} \\
& -829854687438 c_{2}^{2} d^{4} a_{2} b^{2}+829854687438 a_{2}^{2} b^{4} c_{2} d^{2}-16524095013 a_{2} b^{2} c_{2}^{4} d^{8} \\
& -1198678992966 a_{2} a b^{3} c_{2} c d^{3}+3077 a_{2}^{2} a b^{5} c_{2}^{8} c d^{17}+3077 a_{2}^{8} a b^{17} c_{2}^{2} c d^{5}+765 a_{2}^{4} a b^{9} c_{2}^{8} c d^{17} \\
& +282429536481 a b c d-3008487501 c_{2}^{3} d^{6} a_{2}^{2} b^{4}-5661 c_{2}^{7} d^{14} a_{2}^{6} b^{12} \\
& +16524095013 a_{2}^{4} b^{8} c_{2} d^{2}+2142 a_{2}^{8} b^{16} c_{2}^{7} d^{14}-2142 a_{2}^{7} b^{14} c_{2}^{8} d^{16}+a_{2}^{8} a b^{17} c_{2}^{8} c d^{17} \\
& -3094 a_{2}^{7} a b^{15} c_{2}^{7} c d^{15}+230516343504 c_{2}^{4} c d^{9} a_{2}^{2} a b^{5}+14701702 c_{2}^{7} c d^{15} a_{2} a b^{3} \\
& +1921729062 c_{2}^{5} c d^{11} a_{2}^{3} a b^{7}+3630060354 c_{2}^{6} c d^{13} a_{2}^{2} a b^{5}+5609286 c_{2}^{7} c d^{15} a_{2}^{3} a b^{7} \\
& +4089169494 c_{2}^{5} c d^{11} a_{2} a b^{3}+3630060354 c_{2}^{2} c d^{5} a_{2}^{6} a b^{13}+728053975242 a_{2}^{3} a b^{7} c_{2} c d^{3} \\
& +14701702 a_{2}^{7} a b^{15} c_{2} c d^{3}+5609286 a_{2}^{7} a b^{15} c_{2}^{3} c d^{7}+4089169494 a_{2}^{5} a b^{11} c_{2} c d^{3} \\
& +316208976 a_{2}^{6} a b^{13} c_{2}^{4} c d^{9}+6619358 a_{2}^{6} a b^{13} c_{2}^{6} c d^{13}+1369962 a_{2}^{7} a b^{15} c_{2}^{5} c d^{11}
\end{aligned}
$$


$+230516343504 a_{2}^{4} a b^{9} c_{2}^{2} c d^{5}+728053975242 c_{2}^{3} c d^{7} a_{2} a b^{3}+1921729062 c_{2}^{3} c d^{7} a_{2}^{5} a b^{11}$

$+25749161622 c_{2}^{4} c d^{9} a_{2}^{4} a b^{9}+291817274 c_{2}^{5} c d^{11} a_{2}^{5} a b^{11}+212734792746 c_{2}^{3} c d^{7} a_{2}^{3} a b^{7}$

$+316208976 c_{2}^{6} c d^{13} a_{2}^{4} a b^{9}+1369962 c_{2}^{7} c d^{15} a_{2}^{5} a b^{11}+3517798234878 a_{2}^{2} a b^{5} c_{2}^{2} c d^{5}$

$+765 a_{2}^{8} a b^{17} c_{2}^{4} c d^{9}+51 a_{2}^{8} a b^{17} c_{2}^{6} c d^{13}+51 a_{2}^{6} a b^{13} c_{2}^{8} c d^{17}-c_{2}^{9} d^{18}+a_{2}^{9} b^{18}=0$,

where

$$
a_{2}=\frac{\varphi^{4}(-q)}{\varphi^{4}\left(-q^{3}\right)} \quad \text { and } \quad c_{2}=\frac{\varphi^{4}\left(-q^{17}\right)}{\varphi^{4}\left(-q^{51}\right)}
$$

Using the equation (2.8) in the equation (3.8), we obtain the equation (3.6).

Remark 2. The equation (3.6) holds for

$$
P=\frac{\varphi(q) \varphi\left(q^{17}\right)}{\varphi\left(q^{3}\right) \varphi\left(q^{51}\right)} \quad \text { and } \quad Q=\frac{\varphi(q) \varphi\left(q^{51}\right)}{\varphi\left(q^{3}\right) \varphi\left(q^{17}\right)} .
$$

Theorem 3.3. If $P=\frac{\psi(q) \psi\left(q^{19}\right)}{q^{5} \psi\left(q^{3}\right) \psi\left(q^{57}\right)}$ and $Q=\frac{\psi(q) \psi\left(q^{57}\right)}{q^{-9 / 2} \psi\left(q^{3}\right) \psi\left(q^{19}\right)}$, then

$$
\begin{aligned}
& Q^{10}-\frac{1}{Q^{10}}+\left(P^{9}+\frac{3^{9}}{P^{9}}\right)+19\left\{26\left(Q^{8}-\frac{1}{Q^{8}}\right)+135\left(Q^{6}-\frac{1}{Q^{6}}\right)\right. \\
& +1916\left(Q^{4}-\frac{1}{Q^{4}}\right)+1144\left(Q^{2}-\frac{1}{Q^{2}}\right)-\left(P^{7}+\frac{3^{7}}{P^{7}}\right)\left[\left(Q^{2}-\frac{1}{Q^{2}}\right)-4\right] \\
& -5\left(P^{6}+\frac{3^{6}}{P^{6}}\right)\left[\left(Q^{2}-\frac{1}{Q^{2}}\right)\right]+3\left(P^{5}+\frac{3^{5}}{P^{5}}\right)\left[2\left(Q^{4}+\frac{1}{Q^{4}}\right)\right. \\
& \left.-14\left(Q^{2}+\frac{1}{Q^{2}}\right)+11\right]+2\left(P^{4}+\frac{3^{4}}{P^{4}}\right)\left[26\left(Q^{4}-\frac{1}{Q^{4}}\right)-\left(Q^{2}-\frac{1}{Q^{2}}\right)\right] \\
& -5\left(P^{2}+\frac{3^{2}}{P^{2}}\right)\left[13\left(Q^{6}-\frac{1}{Q^{6}}\right)-4\left(Q^{4}-\frac{1}{Q^{4}}\right)+104\left(Q^{2}-\frac{1}{Q^{2}}\right)\right] \\
& -\left(P^{3}+\frac{3^{3}}{P^{3}}\right)\left[12\left(Q^{6}+\frac{1}{Q^{6}}\right)-65\left(Q^{4}+\frac{1}{Q^{4}}\right)-591\right. \\
& \left.+200\left(Q^{2}+\frac{1}{Q^{2}}\right)\right]+3\left(P+\frac{3}{P}\right)\left[2\left(Q^{8}+\frac{1}{Q^{8}}\right)-29\left(Q^{6}+\frac{1}{Q^{6}}\right)\right. \\
& \left.+7\left(Q^{4}+\frac{1}{Q^{4}}\right)-504\left(Q^{2}+\frac{1}{Q^{2}}\right)+697\right]=0
\end{aligned}
$$

The proof of the equation (3.9) is similar to the proof of the equation (3.6) except in the place of the result (2.4), the result (2.5) is used.

Remark 3. The equation (3.9) holds for

$$
P=\frac{\varphi(q) \varphi\left(q^{19}\right)}{\varphi\left(q^{3}\right) \varphi\left(q^{57}\right)} \quad \text { and } \quad Q=\frac{\varphi(q) \varphi\left(q^{57}\right)}{\varphi\left(q^{3}\right) \varphi\left(q^{19}\right)} .
$$




\section{General formulas for the explicit evaluations of ratios of Ramanujan's theta functions}

In this section, we establish some general formulas for the explicit evaluations of ratios of Ramanujan's theta functions.

Theorem 4.1. If $X=h_{3, n}$ and $Y=h_{3,81 n}$, then

$$
\begin{aligned}
& \frac{Y^{5}}{X^{5}}+9\left(\frac{Y^{3}}{X^{3}}-\frac{Y^{4}}{X^{4}}\right)+9\left(\frac{5 Y}{X}+\frac{9 X}{Y}\right)+27\left(Y^{4}+\frac{1}{X^{4}}\right) \\
& +9\left(Y^{2}+3 X^{2}\right)\left(X Y+\frac{1}{X^{3} Y^{3}}\right) \\
= & 135+3\left(\frac{Y^{2}}{X^{2}}+\frac{9 X^{2}}{Y^{2}}\right)+9\left(X^{4} Y^{4}+\frac{1}{X^{4} Y^{4}}\right)+27\left(X^{2} Y^{2}+\frac{1}{X^{2} Y^{2}}\right) .
\end{aligned}
$$

Proof. Employing the equation (3.1) along with the equation (1.5) with $k=3$, we obtain the equation (4.1).

Corollary 4.1. We have

$$
\begin{aligned}
h_{3,9} & =\frac{\sqrt[3]{4}-\sqrt[3]{2}+1}{\sqrt{3}}, \\
h_{3,1 / 9} & =\frac{1+\sqrt[3]{2}}{\sqrt{3}}, \\
l_{3,9} & =\frac{(\sqrt[3]{2}+1)^{2}}{\sqrt{3}}, \\
l_{3,1 / 9} & =\frac{(\sqrt[3]{4}-1)}{\sqrt{3}} .
\end{aligned}
$$

Proofs of (4.2) and (4.3). Putting $n=1 / 9$ in the equation (3.1) and then using the equation (2.9), we deduce that

$$
\left(h_{3,9}^{3}+h_{3,9}^{2} \sqrt{3}+3 h_{3,9}+\sqrt{3}\right)\left(-h_{3,9}^{3}+h_{3,9}^{2} \sqrt{3}-3 h_{3,9}+\sqrt{3}\right)\left(h_{3,9}^{4}-6 h_{3,9}^{2}+3\right)^{2}=0 .
$$

Since $0<h_{3,9}<1$, we find that

$$
h_{3,9}^{3}-h_{3,9}^{2} \sqrt{3}+3 h_{3,9}-\sqrt{3}=0 .
$$

On solving the above equation, we arrive at the equations (4.2) and (4.3).

Proofs of (4.4) and (4.5). Using the equations (2.8), (4.2) and (4.3), we arrive at the equations (4.4) and (4.5).

Remark 4. Different proofs of the equations (4.2) and (4.4) can be found in [4], [15] and [16]. 
Theorem 4.2. If $X=h_{3, n} h_{3,289 n}$ and $Y=\frac{h_{3, n}}{h_{3,289 n}}$, then

$$
\begin{aligned}
& Y^{9}-\frac{1}{Y^{9}}+34\left(Y^{8}+\frac{1}{Y^{8}}\right)+272\left(Y^{7}-\frac{1}{Y^{7}}\right)+238\left(Y^{6}+\frac{1}{Y^{6}}\right) \\
& -595\left(Y^{5}-\frac{1}{Y^{5}}\right)-510\left(Y^{4}+\frac{1}{Y^{4}}\right)+16303\left(Y^{3}-\frac{1}{Y^{3}}\right) \\
& -5202\left(Y^{2}+\frac{1}{Y^{2}}\right)-26911\left(Y-\frac{1}{Y}\right)+3^{4}\left(X^{8}+\frac{1}{X^{8}}\right)+20230 \\
= & 51\left\{( X ^ { 2 } + \frac { 1 } { X ^ { 2 } } ) \left[7\left(Y^{6}+\frac{1}{Y^{6}}\right)+28\left(Y^{5}-\frac{1}{Y^{5}}\right)+34\left(Y^{4}+\frac{1}{Y^{4}}\right)\right.\right. \\
& \left.-168\left(Y^{3}-\frac{1}{Y^{3}}\right)+160\left(Y^{2}+\frac{1}{Y^{2}}\right)+378\left(Y-\frac{1}{Y}\right)-210\right] \\
& -3\left(X^{4}+\frac{1}{X^{4}}\right)\left[5\left(Y^{4}+\frac{1}{Y^{4}}\right)+21\left(Y^{3}-\frac{1}{Y^{3}}\right)-35\left(Y^{2}+\frac{1}{Y^{2}}\right)\right. \\
& \left.\left.-14\left(Y_{-} \frac{1}{Y}\right)-30\right]-9\left(X^{6}+\frac{1}{X^{6}}\right)\left[\left(Y^{2}+\frac{1}{Y^{2}}\right)-2\left(Y-\frac{1}{Y}\right)-2\right]\right\} .
\end{aligned}
$$

Proof. Employing the equation (3.6) along with the equation (1.5) with $k=3$ and Remark 2, we obtain the equation (4.8).

Corollary 4.2. We have

$$
\begin{aligned}
h_{3,17}^{2} & =\frac{2+\sqrt{17}-(142+34 \sqrt{17})^{1 / 3}+(2+2 \sqrt{17})^{1 / 3}}{3}, \\
h_{3,1 / 17}^{2} & =\frac{\sqrt{17}-2+(142-34 \sqrt{17})^{1 / 3}+(-2+2 \sqrt{17})^{1 / 3}}{3}, \\
l_{3,17}^{2} & =4+\sqrt{17}+2(29+7 \sqrt{17})^{1 / 3}+2(37+9 \sqrt{17})^{1 / 3}, \\
l_{3,1 / 17}^{2} & =-4+\sqrt{17}-2(29-7 \sqrt{17})^{1 / 3}+2(-37+9 \sqrt{17})^{1 / 3} .
\end{aligned}
$$

Proofs of (4.9) and (4.10). Putting $n=1 / 17$ in the equation (4.8) and then using the equation $(2.9)$, we find that

$$
\begin{aligned}
& \left(h_{3,17}^{12}-4 h_{3,17}^{10}+5 h_{3,17}^{8}+24 h_{3,17}^{6}-5 h_{3,17}^{4}-4 h_{3,17}^{2}-1\right) \\
& \quad \times\left(h_{3,17}^{4}+h_{3,17}^{2}-1\right)^{2}\left(h_{3,17}^{8}-16 h_{3,17}^{6}-22 h_{3,17}^{4}+16 h_{3,17}^{2}+1\right)^{2}=0 .
\end{aligned}
$$

We observe that the first factor of the equation (4.13) vanishes for the specific value of $q=e^{-\pi \sqrt{17 / 3}}$, but the other two factors does not vanish. Since $0<h_{3,17}<1$, hence we deduce that

$$
x^{3}+8 x-4 x^{2}+16=0,
$$

where $x=h_{3,17}^{2}-\frac{1}{h_{3,17}^{2}}$. 
On solving the above equation, we find that

$$
h_{3,17}^{2}-\frac{1}{h_{3,17}^{2}}=\frac{2}{3}\left[(-37+9 \sqrt{17})^{1 / 3}-(37+9 \sqrt{17})^{1 / 3}+2\right] .
$$

On solving the above equation, we arrive at the equations (4.9) and (4.10).

Proofs of (4.11) and (4.12). By using the equation (2.8) along with the equations (4.9) and (4.10) respectively, we arrive at the equations (4.11) and (4.12).

Theorem 4.3. If $X=h_{3, n} h_{3,361 n}$ and $Y=\frac{h_{3, n}}{h_{3,361 n}}$, then

$$
\begin{aligned}
& Y^{10}-\frac{1}{Y^{10}}+3^{4} \sqrt{3}\left(X^{9}+\frac{1}{X^{9}}\right)+19\left\{1916\left(Y^{4}-\frac{1}{Y^{4}}\right)+135\left(Y^{6}-\frac{1}{Y^{6}}\right)\right. \\
& +26\left(Y^{8}-\frac{1}{Y^{8}}\right)+1144\left(Y^{2}-\frac{1}{Y^{2}}\right)-3^{3} \sqrt{3}\left(X^{7}+\frac{1}{X^{7}}\right)\left[\left(Y^{2}-\frac{1}{Y^{2}}\right)-4\right] \\
& -135\left(X^{6}+\frac{1}{X^{6}}\right)\left[\left(Y^{2}-\frac{1}{Y^{2}}\right)\right]+27 \sqrt{3}\left(X^{5}+\frac{1}{X^{5}}\right)\left[2\left(Y^{4}+\frac{1}{Y^{4}}\right)\right. \\
& \left.-14\left(Y^{2}+\frac{1}{Y^{2}}\right)+11\right]+18\left(X^{4}+\frac{1}{X^{4}}\right)\left[26\left(Y^{4}-\frac{1}{Y^{4}}\right)-\left(Y^{2}-\frac{1}{Y^{2}}\right)\right] \\
& -15\left(X^{2}+\frac{1}{X^{2}}\right)\left[13\left(Y^{6}-\frac{1}{Y^{6}}\right)-4\left(Y^{4}-\frac{1}{Y^{4}}\right)+104\left(Y^{2}-\frac{1}{Y^{2}}\right)\right] \\
& +3 \sqrt{3}\left(X^{3}+\frac{1}{X^{3}}\right)\left[12\left(Y^{6}+\frac{1}{Y^{6}}\right)-65\left(Y^{4}+\frac{1}{Y^{4}}\right)-591\right. \\
& \left.\left.+7\left(Y^{4}+\frac{1}{Y^{4}}\right)-504\left(Y^{2}+\frac{1}{Y^{2}}\right)+697\right]\right\}=0 .
\end{aligned}
$$

Proof. Employing the equation (3.9) along with the equation (1.5) with $k=3$ and Remark 3, we obtain the equation (4.16).

Corollary 4.3. We have

$$
\begin{aligned}
h_{3,19}^{4} & =(26-15 \sqrt{3})(2 \sqrt{19}+5 \sqrt{3}), \\
h_{3,1 / 19}^{4} & =(26+15 \sqrt{3})(2 \sqrt{19}-5 \sqrt{3}), \\
l_{3,19}^{4} & =(26+15 \sqrt{3})(2 \sqrt{19}+5 \sqrt{3}), \\
l_{3,1 / 19}^{4} & =(26-15 \sqrt{3})(2 \sqrt{19}-5 \sqrt{3}) .
\end{aligned}
$$


Proofs of (4.17) and (4.18). Putting $n=1 / 19$ in the equation (4.16) and then using the equation $(2.9)$, we find that

$$
\begin{aligned}
& \left(h_{3,19}^{8}+(450+260 \sqrt{3}) h_{3,19}^{4}-1351-780 \sqrt{3}\right) \\
\times & \left(-h_{3,19}^{8}-(4-4 \sqrt{3}) h_{3,19}^{4}+2-\sqrt{3}\right)^{2} \\
\times & \left(-h_{3,19}^{8}-(18-12 \sqrt{3}) h_{3,19}^{4}+7-4 \sqrt{3}\right)^{2}=0 .
\end{aligned}
$$

Since $0<h_{3,19}<1$, we deduce that

$$
h_{3,19}^{8}+(450-260 \sqrt{3}) h_{3,19}^{4}-1351+780 \sqrt{3}=0 .
$$

Solving the above equation, we obtain (4.17) and (4.18).

Proofs of (4.19) and (4.20). Using the equations (2.8), (4.17) and (4.18), we arrive at the equations (4.19) and (4.20).

\section{Modular relations for Ramanujan's cubic continued fraction}

In this section, by using the modular equations established in Section 3 we find modular relations connecting $V(q)$ with other three continued fractions $V\left(q^{9}\right)$, $V\left(q^{17}\right)$ and $V\left(q^{19}\right)$.

Theorem 5.1. If $v=V(q)$ and $w=V\left(q^{9}\right)$, then

$$
\begin{aligned}
& w^{9}-\left(36 v^{3}+256 v^{9}-5+288 v^{6}\right) w^{8}-\left(90 v^{3}-640 v^{9}-576 v^{6}-10\right) w^{7} \\
& -\left(648 v^{6}+640 v^{9}+81 v^{3}-11\right) w^{6}-\left(-10-352 v^{9}+72 v^{3}-396 v^{6}\right) w^{5} \\
& -\left(144 v^{6}-11+99 v^{3}+160 v^{9}\right) w^{4}-\left(-88 v^{9}+81 v^{3}-10-81 v^{6}\right) w^{3} \\
& -\left(40 v^{9}+45 v^{6}-5+36 v^{3}\right) w^{2}-\left(-9 v^{6}-1-10 v^{9}+9 v^{3}\right) w-v^{9}=0 .
\end{aligned}
$$

Proof. Using the Remark 1, we deduce that

$$
\begin{aligned}
& -1944 w^{9} Q^{2} v^{6}+396 w^{9} Q^{2} v^{3}-432 w^{12} Q^{2} v^{6}-120 w^{12} Q^{2} v^{3}-4848 v^{12} Q^{2} w^{6} \\
& -468 v^{9} Q^{2} w^{3}-232 v^{12} Q^{2} w^{3}-9288 v^{9} Q^{2} w^{6}-15808 v^{12} w^{9} Q^{2}-18144 v^{9} w^{9} Q^{2} \\
& -256 Q^{2} v^{12} w^{12}-576 Q^{2} v^{9} w^{12}+P^{2}+423 Q^{2} v^{3} w^{6}-93 Q^{2} v^{3} w^{3}-4050 Q^{2} v^{6} w^{6} \\
& -351 Q^{2} v^{6} w^{3}+5064 P^{2} v^{6} w^{9}-16832 P^{2} v^{12} w^{9}-1352 P^{2} v^{12} w^{3}-10016 P^{2} v^{9} w^{9} \\
& -12648 P^{2} v^{9} w^{6}-380 P^{2} w^{9} v^{3}+40 P^{2} w^{12} v^{3}+240 P^{2} w^{12} v^{6}+256 P^{2} v^{12} w^{12} \\
& +448 P^{2} v^{9} w^{12}+241 P^{2} v^{3} w^{3}-165 P^{2} v^{3} w^{6}+2898 P^{2} v^{6} w^{6}-11856 P^{2} v^{12} w^{6} \\
& -2204 P^{2} v^{9} w^{3}-14 P^{2} v^{3}+4 P^{2} w^{3}+6 P^{2} w^{6}+51 P^{2} v^{6}-1875 P^{2} v^{6} w^{3}-v^{12} Q^{2} \\
& +4 P^{2} w^{9}-23 P^{2} v^{12}+18 Q^{2} w^{3}+27 w^{6} Q^{2}-9 w^{12} Q^{2}-20 P^{2} v^{9}+P^{2} w^{12}=0
\end{aligned}
$$


where

$$
P=\frac{\psi(q)}{q^{1 / 4} \psi\left(q^{3}\right)} \quad \text { and } \quad Q=\frac{\psi\left(q^{9}\right)}{q^{9 / 4} \psi\left(q^{27}\right)} .
$$

By collecting the terms containing $P^{2}$ on one side of the equation (5.2), then squaring both sides along with the equation (2.7), we find that

$$
\begin{aligned}
& \left(-10 w^{3}+81 v^{3} w^{3}-11 w^{6}-w+648 v^{6} w^{6}+81 v^{3} w^{6}-81 v^{6} w^{3}-5 w^{2}\right. \\
& -88 v^{9} w^{3}+640 v^{9} w^{6}+9 v^{3} w+36 v^{3} w^{2}+99 v^{3} w^{4}+v^{9}-w^{9}-11 w^{4}-5 w^{8} \\
& -10 w^{5}+45 v^{6} w^{2}+288 v^{6} w^{8}+72 v^{3} w^{5}-396 v^{6} w^{5}+36 w^{8} v^{3}-576 v^{6} w^{7} \\
& +144 v^{6} w^{4}-9 w v^{6}+90 w^{7} v^{3}-10 w^{7}-10 v^{9} w+40 v^{9} w^{2}+160 v^{9} w^{4} \\
& \left.+256 v^{9} w^{8}-352 v^{9} w^{5}-640 v^{9} w^{7}\right) \\
& \times\left(-5 w^{3}+81 v^{3} w^{3}+35 w^{6}+4698 v^{6} w^{6}-567 v^{3} w^{6}-324 v^{6} w^{3}+w^{2}\right. \\
& -23409 v^{12} w^{6}-111 v^{9} w^{3}-13851 v^{6} w^{9}+110808 v^{12} w^{9}+119376 v^{9} w^{9} \\
& +567 v^{12} w^{3}-16023 v^{9} w^{6}-23409 w^{12} v^{6}+567 w^{12} v^{3}-729 w^{9} v^{3} \\
& +300672 v^{12} w^{12}+128184 v^{9} w^{12}-18 v^{3} w^{2}-189 v^{3} w^{4}-14 w^{12}+55 w^{9}+15 w^{4} \\
& -14 w^{8}-28 w^{5}+99 v^{6} w^{2}-198 v^{6} w^{8}+369 v^{3} w^{5}-207 w^{8} v^{3}+594 v^{6} w^{7} \\
& -54 v^{6} w^{4}+621 w^{7} v^{3}-21 w^{7}-7104 v^{9} w^{15}+165888 v^{12} w^{15}-46656 v^{15} w^{9} \\
& -28160 v^{18} w^{9}+290304 v^{15} w^{12}+163840 v^{18} w^{15}+331776 v^{15} w^{15}-896 v^{18} w^{6} \\
& +143360 v^{18} w^{12}-4536 v^{15} w^{6}+648 v^{3} w^{15}+648 v^{15} w^{3}-4536 v^{6} w^{15} \\
& +224 v^{18} w^{3}-28 w^{15}+w^{18}+v^{18}+55 w^{11}+35 w^{14}-5 w^{17}-21 w^{13}-72 w^{10} \\
& +15 w^{16}-1557 v^{3} w^{11}-927 v^{3} w^{14}+36 v^{3} w^{17}+459 v^{3} w^{13}+1539 v^{3} w^{10} \\
& -270 v^{3} w^{16}+23508 v^{6} w^{11}+22716 v^{6} w^{14}+288 v^{6} w^{17}-3618 v^{6} w^{13}-810 v^{6} w^{10} \\
& -2160 v^{6} w^{16}+v^{9} w-137 v^{9} w^{2}+4121 v^{9} w^{4}+24664 v^{9} w^{8}-49 v^{9} w^{5} \\
& -26888 v^{9} w^{7}-107552 v^{9} w^{11}-131872 v^{9} w^{14}+256 v^{9} w^{17}-784 v^{9} w^{13} \\
& -49328 v^{9} w^{10}+17536 v^{9} w^{16}-9 v^{12} w-135 v^{12} w^{2}+5679 v^{12} w^{4}-3240 v^{12} w^{8} \\
& +1809 v^{12} w^{5}-47016 v^{12} w^{7}-19008 v^{12} w^{11}-13824 v^{12} w^{14}+150912 v^{12} w^{13} \\
& -3168 v^{12} w^{10}+101376 v^{12} w^{16}+9 v^{15} w+135 v^{15} w^{2}+1854 v^{15} w^{4}-49248 v^{15} w^{8} \\
& +1836 v^{15} w^{5}-24912 v^{15} w^{7}+158976 v^{15} w^{11}+387072 v^{15} w^{14}+377856 v^{15} w^{13} \\
& +26496 v^{15} w^{10}+147456 v^{15} w^{16}+10 v^{18} w+60 v^{18} w^{2}+560 v^{18} w^{4}-18432 v^{18} w^{8} \\
& +672 v^{18} w^{5}-7040 v^{18} w^{7}+43008 v^{18} w^{11}+245760 v^{18} w^{14}+229376 v^{18} w^{13} \\
& \left.-14336 v^{18} w^{10}+65536 v^{18} w^{16}-1179 v^{6} w^{5}\right)=0 .
\end{aligned}
$$

From the definitions of $v$ and $w$, we have $v=o\left(q^{1 / 3}\right)$ and $w=o\left(q^{3}\right)$ as $q \rightarrow 0$, it can be seen that the first factor of the equation (5.3) vanishes for $q$ sufficiently small; whereas the second factor does not vanish. Thus by the Identity Theorem, first factor vanishes identically. This completes the proof. 
Theorem 5.2. If $v=V(q)$ and $w=V\left(q^{17}\right)$, then

$$
\begin{aligned}
& w^{18}-w v\left[(2 v w)^{16}+1\right]+v^{18}+17\left\{\left(1-5 v^{3}+5 v^{6}+9 v^{9}-v^{12}\right) v^{4} w\right. \\
& +\left(16384 v^{15}+36864 v^{12}+12288 v^{9}-7488 v^{6}+712 v^{3}-1\right) v w^{16} \\
& +8\left(79-1059 v^{3}+2952 v^{6}+1024 v^{9}-3072 v^{12}\right) v^{3} w^{15}+(178 \\
& \left.-8192 v^{15}-75776 v^{12}-50560 v^{9}+15024 v^{6}-2002 v^{3}\right) v^{2} w^{14} \\
& +\left(36864 v^{15}+33792 v^{12}-13120 v^{9}-9560 v^{6}+1001 v^{3}+9\right) v w^{13} \\
& +\left(8192 v^{12}+38016 v^{9}+17496 v^{6}-10177 v^{3}+1059\right) v^{3} w^{12} \\
& +2\left(117-1195 v^{3}+3016 v^{6}-19328 v^{9}-25280 v^{12}-2560 v^{15}\right) v^{2} w^{11} \\
& +\left(12288 v^{15}-13120 v^{12}-26784 v^{9}-3016 v^{6}+939 v^{3}+5\right) v w^{10} \\
& +\left(369-2187 v^{3}-11536 v^{6}+17496 v^{9}+23616 v^{12}\right) v^{3} w^{9}+2\left(205 v^{3}\right. \\
& \left.-320 v^{15}+7512 v^{12}+3016 v^{9}-3348 v^{6}+24\right) v^{2} w^{8}-\left(7488 v^{15}+9560 v^{12}\right. \\
& \left.+3016 v^{9}+2416 v^{6}-395 v^{3}+5\right) v w^{7}-\left(8472 v^{12}+10177 v^{9}+2187 v^{6}+16\right. \\
& \left.-594 v^{3}\right) v^{3} w^{6}+2\left(72 v^{15}-1001 v^{12}-1195 v^{9}+205 v^{6}+66 v^{3}-9\right) v^{2} w^{5} \\
& +\left(712 v^{15}+1001 v^{12}+939 v^{9}+395 v^{6}-74 v^{3}+1\right) v w^{4}+\left(632 v^{12}+1059 v^{9}\right. \\
& \left.+369 v^{6}-16 v^{3}-6\right) v^{3} w^{3}+2\left(9(2 v)^{3}+1-5(2 v)^{6}-(2 v)^{12}-5(2 v)^{9}\right) v^{2} w^{17} \\
& \left.+\left(2 v^{15}+178 v^{12}+234 v^{9}+48 v^{6}-18 v^{3}+1\right) v^{2} w^{2}\right\}=0
\end{aligned}
$$

The proof of the equation (5.4) is similar to the proof of the equation (5.1), except in the place of the result (3.1), the result (3.6) is used.

Remark 5. A different proof of the equation (5.4) can be found in [8].

Theorem 5.3. If $v=V(q)$ and $w=V\left(q^{19}\right)$, then

$$
\begin{aligned}
& v^{20}+w^{20}-w v\left\{(2 w v)^{18}+1\right\}+19\left\{4 \left(-8192 w^{15}-6144 w^{12}-1280 w^{9}+22 w^{3}-1\right.\right. \\
& \left.+144 w^{6}\right) w v^{19}+2 w^{2} v^{18}\left[53-2^{4}\left(4096 w^{15}+8192 w^{12}+3072 w^{9}-944 w^{6}+77 w^{3}\right)\right] \\
& -4 w^{3} v^{17}\left(243-2492 w^{3}-8608 w^{6}+94464 w^{9}+133120 w^{12}+32768 w^{15}\right)+w v^{16} \\
& \times\left(-11+2124 w^{3}-13096 w^{6}+57728 w^{9}-463872 w^{12}-569344 w^{15}-32768 w^{18}\right) \\
& -4 w^{2} v^{15}\left(133120 w^{15}+152832 w^{12}+12496 w^{9}-6266 w^{6}+1077 w^{3}-77\right) \\
& -2 w^{3} v^{14}\left(131072 w^{15}+305664 w^{12}+229568 w^{9}+40136 w^{6}-15237 w^{3}+623\right) \\
& +w v^{13}\left(9+1637 w^{3}-852 w^{6}-64704 w^{9}-505600 w^{12}-463872 w^{15}-24576 w^{18}\right) \\
& +w^{2} v^{12}\left(472-3133 w^{3}+17721 w^{6}-66584 w^{9}-459136 w^{12}-377856 w^{15}\right) \\
& +2 w^{3} v^{11}\left(269+5017 w^{3}-57124 w^{6}-33292 w^{9}-24992 w^{12}-49152 w^{15}\right) \\
& +2 w v^{10}\left(5+451 w^{3}+4044 w^{6}-55316 w^{9}-32352 w^{12}+28864 w^{15}-2560 w^{18}\right) \\
& +w^{2} v^{9}\left(192-781 w^{3}+8323 w^{6}-114248 w^{9}-80272 w^{12}+34432 w^{15}\right) \\
& +w^{3} v^{8}\left(30208 w^{15}+25064 w^{12}+17721 w^{9}+8323 w^{6}-7174 w^{3}+738\right)
\end{aligned}
$$




$$
\begin{aligned}
& +w v^{7}\left(576 w^{18}-13096 w^{15}-852 w^{12}+8088 w^{9}-7900 w^{6}+906 w^{3}-6\right) \\
& +w^{2} v^{6}\left(9968 w^{15}+30474 w^{12}+10034 w^{9}-7174 w^{6}+1194 w^{3}-64\right) \\
& -w^{3} v^{5}\left(2464 w^{15}+4308 w^{12}+3133 w^{9}+781 w^{6}-1194 w^{3}+130\right) \\
& +w v^{4}\left(88 w^{18}+2124 w^{15}+1637 w^{12}+902 w^{9}+906 w^{6}-139 w^{3}+1\right) \\
& +w^{2} v^{3}\left(4-130 w^{3}+738 w^{6}+538 w^{9}-1246 w^{12}-972 w^{15}\right) \\
& +w^{3} v^{2}\left(4-64 w^{3}+192 w^{6}+472 w^{9}+308 w^{12}+106 w^{15}\right) \\
& \left.+w^{4} v\left(9 w^{9}-11 w^{12}+1-4 w^{15}-6 w^{3}+10 w^{6}\right)\right\}=0
\end{aligned}
$$

The proof of the equation (5.5) is similar to the proof of the equation (5.1), except in the place of the result (3.1), the result (3.9) is used.

Acknowledgement. The authors are thankful to the referee for his/her useful comments.

\section{References}

[1] C. Adiga, Taekyun Kim, M. S. Mahadeva Naika and H. S. Madhusudhan, On Ramnujan's cubic continued fraction and explicit evaluations of thetafunctions, Indian J. Pure Appl. Math. 35(9) (2004), 1047-1062.

[2] C. Adiga, K. R. Vasuki, and M. S. Mahadeva Naika, Some new explicit evaluations of Ramanujan's cubic continued fraction, New Zealand J. Math. 31 (2002), 1-6.

[3] N. D. Baruah, Modular equations for Ramanujan's cubic continued fraction, J. Math. Anal. Appl. 268 (2002), 244-255.

[4] N. D. Baruah and N. Saikia, Two parameters for Ramanujan's theta-functions and their explicit values, Rocky Mountain J. Math. 37(6) (2007), 1747-1790.

[5] B. C. Berndt, Ramanujan's Notebooks, Part III, Springer-Verlag, New York, 1991.

[6] S. Bhargava, C. Adiga and M. S. Mahadeva Naika, A new class of modular equations akin to Ramanujan's $P-Q$ eta-function identities and some evaluations there from, Adv. Stud. Contemp. Math. 5(1) (2002), 37-48.

[7] H. H. Chan, On Ramanujan's cubic continued fraction, Acta Arith. 73 (1995), 343-355.

[8] B. Cho, J. K. Koo and Y. K. Park, On the Ramanujan's cubic continued fraction as modular function, Tohoku Math. J. 62(4) (2010), 579-603.

[9] M. S. Mahadeva Naika, Some theorems on Ramanujan's cubic continued fraction and related identities, Tamsui Oxf. J. Math. Sci. 24(3) (2008), 243-256.

[10] M. S. Mahadeva Naika, S. Chandankumar and K. Sushan Bairy, Modular equations for the ratios of Ramnujan's theta function $\psi$ and evaluations, New Zealand J. Math. 40 (2010), 33-48.

[11] M. S. Mahadeva Naika, M. C. Maheshkumar and K. Sushan Bairy, General formulas for explicit evaluations of Ramanujan's cubic continued fraction, Kyungpook Math. J. 49(3) (2009), 435-450. 
[12] M. S. Mahadeva Naika, K. Sushan Bairy and M. Manjunatha, Some new modular equations of degree four and their explicit evaluations, Eur. J. Pure Appl. Math. 3(6) (2010), 924-947.

[13] S. Ramanujan, Notebooks (2 volumes), Tata Institute of Fundamental Research, Bombay, 1957.

[14] S. Ramanujan, The lost notebook and other unpublished papers, Narosa, New Delhi, 1988.

[15] J. Yi, Theta-function identities and the explicit formulas for theta-function and their applications, J. Math. Anal. Appl. 292 (2004), 381-400.

[16] J. Yi, Yang Lee and Dae Hyun Paek, The explicit formulas and evaluations of Ramanujan's theta-function $\psi$, J. Math. Anal. Appl. 321 (2006), 157-181.

[17] J. Yi and Hyo Seob Sim, Some new modular equations and their applications, J. Math. Anal. Appl. 319 (2006), 531-546.

Addresses: M.S. Mahadeva Naika, S. Chandankumar, K. Sushan Bairy: Department of Mathematics, Bangalore University, Central College Campus, Bangalore-560 001, Karnataka, India.

E-mail: msmnaika@rediffmail.com, chandan.s17@gmail.com, ksbairy@rediffmail.com

Received: 3 September 2010; revised: 19 April 2011 